\title{
Using the Autism-Spectrum Quotient to Discriminate Autism Spectrum Disorder from ADHD in Adult Patients With and Without Comorbid Substance Use Disorder
}

\author{
Bram B. Sizoo • Wim van den Brink • Marielle Gorissen-van Eenige • \\ Maarten W. Koeter · Patricia J. M. van Wijngaarden-Cremers · \\ Rutger Jan van der Gaag
}

Published online: 25 April 2009

(c) The Author(s) 2009. This article is published with open access at Springerlink.com

\begin{abstract}
It is unknown whether the Autism-spectrum quotient (AQ) can discriminate between Autism Spectrum Disorder (ASD) and Attention Deficit and Hyperactivity Disorder (ADHD) with or without comorbid Substance Use Disorder (SUD). ANOVA's were used to analyse the mean AQ (sub)scores of 129 adults with ASD or ADHD. We applied receiver operating characteristic (ROC) computations to assess discriminant power. All but one of the mean AQ (sub)scores were significantly higher for adults with ASD compared to those with ADHD. The SUD status in general was not significantly associated with AQ (sub)scores. On the Social Skills subscale patients with ASD and comorbid SUD showed less impairment than those without
\end{abstract}

B. B. Sizoo $(\varangle)$ · P. J. M. van Wijngaarden-Cremers Department of Developmental Disorders, Dimence Institute of Mental Health, P.O. Box 5003, 7400 GC Deventer, The Netherlands

e-mail: b.sizoo@dimence.nl

B. B. Sizoo · P. J. M. van Wijngaarden-Cremers ·

R. J. van der Gaag

Karakter Department of Child and Adolescent Psychiatry,

University Medical Centre St. Radboud, Nijmegen,

The Netherlands

W. van den Brink - M. W. Koeter

Department of Psychiatry, Academic Medical Center,

University of Amsterdam, Amsterdam, The Netherlands

W. van den Brink - M. W. Koeter

Amsterdam Institute for Addiction Research, Amsterdam,

The Netherlands

M. Gorissen-van Eenige

The American University of Paris, Paris, France

M. Gorissen-van Eenige

TELL Counseling and Assessment, Tokyo, Japan
SUD. The cut-off score 26 yielded $73 \%$ correct classifications. The clinical use of the AQ in differentiating between ASD and ADHD is limited.

Keywords Autism spectrum disorder - ADHD . Substance use disorder · Autism-spectrum quotient

\section{Introduction}

Autism spectrum disorder (ASD) encompasses Autistic Disorder, Asperger's Disorder, and Pervasive Developmental Disorder not otherwise specified (PDD-NOS). The clinical nature of ASD is characterized by a triad of behavioral deficits in the development of (a) reciprocal social interactions and (b) verbal and non-verbal communication, and (c) a restricted patterns of interests and stereotyped behaviors. In addition, many patients with ASD display attention problems (Corbett and Constantine 2006; Courchesne et al. 1989; Wing 1997).

The definition of Attention deficit and hyperactivity disorder (ADHD) centers on the deficit in attention in combination with impulsivity and hyperactivity. The distinction between ASD and ADHD in children can be complicated due to attention symptoms present in both disorders (Goldstein and Volkow 2002; Jensen et al. 1997). It is increasingly recognized that the clinical impairment of developmental disorders like ASD and ADHD may not manifest before adulthood (Faraone et al. 2006; Ketelaars et al. 2008). Particularly in those 'late presentations' it can be very difficult to distinguish between ASD and ADHD, especially when motor hyperactivity is not a prominent ADHD feature (Anckarsäter et al. 2006). Furthermore, classification of ASD and ADHD in adults is complicated because the phrasing of the criteria for these disorders in 
DSM-IV (American Psychiatric Association 2000) and ICD-10 (World Health Organization 2001) is based on school age children. Finally, in adults with ASD and ADHD, Substance use disorder (SUD) may occur as a comorbid condition making the differentiation between ASD and ADHD even more complex (van Wijngaarden et al. 2009).

Baron-Cohen and colleagues developed the Autismspectrum quotient (AQ) as a self-report questionnaire to quantify autistic symptoms in normal intelligent adults. Several publications by different research groups have established the psychometric properties and validity of the instrument (Baron-Cohen et al. 2001; Kurita et al. 2005), although there is still discussion about the cut-off values (Ketelaars et al. 2008; Wakabayashi et al. (2006); Woodbury-Smith et al. 2005). The AQ consists of 50 statements, reflecting personal beliefs, views and preferences. The respondent can agree or disagree with each of these statements using a 4 point Likert scale $(1=$ "definitely agree", 2 = "slightly agree", 3 = "slightly disagree", and $4=$ "definitely disagree") (Woodbury-Smith et al. 2005). The 50 items were divided by the original authors into 5 theoretical subscales of 10 items each: Social skills, Communication, Imagination, Attention to detail, and Attention switching. Recently, Hoekstra et al. (2008) argued for a two factor model, with factor 1 (Attention to detail) consisting of one of the five subscales, and factor 2 (Social interaction) consisting of the remaining four subscales (Hoekstra et al. 2008).

Several studies indicate that the AQ can differentiate reasonably well between ASD and controls in the general population (Baron-Cohen et al. 2001; Wakabayashi et al. 2006). It remains unclear however, to what extend the AQ can differentiate between ASD and other psychopathological conditions. Only one study showed that the AQ can distinguish between ASD and non-ASD conditions in an out-patient setting (Woodbury-Smith et al. 2005). In addition, Cath et al. (2008) indicated that there is a statistically significant difference on two subscales of the AQ scores between patients with Obsessive Compulsive Disorder or Social Anxiety Disorder only and patients with those disorders comorbid with ASD (Cath et al. 2008). The clearest evidence that the AQ differentiates between ASD and different psychopathological conditions, is given by Hoekstra et al. (2008) who showed that the AQ can distinguish between ASD and 'pure' OCD or SAD on the total AQ score and both factor scales (Hoekstra et al. 2008). In short, very little is still known about the discriminating ability of the AQ when it concerns conditions that are difficult to distinguish from ASD, like ADHD (especially when further complicated by comorbid SUD).

The aim of the present study is to determine (a) whether the AQ can discriminate between ASD and ADHD in adults, and (b) whether the discriminating properties of the $\mathrm{AQ}$ are negatively influenced by the presence of a concurrent SUD.

\section{Methods}

Participants

In the course of a broader study design (Sizoo et al. 2009) we presented a battery of instruments, among which the AQ, to 129 participating adults recently diagnosed with ASD or ADHD. Prior approval for the study was obtained from the regional medical ethical committee. A consecutive sample of patients was recruited between January 2006 and June 2007 from two specialized diagnostic clinics for adults with (possible) developmental disorders such as ASD and ADHD.

After having been diagnosed with ASD or ADHD, 191 patients $(n=100$ ASD and $n=91$ ADHD), were informed by their own clinicians about the study, and were asked for permission to be approached by the research team. Seven subjects had been diagnosed with ADHD before being diagnosed with ASD in the centers. Because all ADHD associated symptoms reported by these subjects could - in retrospect-better be explained by ASD, we decided to use only the diagnosis ASD in these patients. Exclusion criteria were: history of comorbid psychotic disorder, bipolar disorder, IQ $<80$, insufficient command of the Dutch language, and uncorrected visual or auditory impairment. Of the 167 patients contacted by our team, 138 agreed to participate after having given informed consent. Of those, 7 did not complete the AQ for various administrative reasons, and 2 were excluded due to a total IQ below 80 . The 29 non-responders were of normal intelligence, and did not differ significantly from the study sample with respect to age and sex distribution. Thus 129 questionnaires could be included for analyses.

The IQ was determined by means of the Wechsler Adult Intelligence Scale-III in 85 patients (Wechsler 1997). For the remainder $(n=44)$, the IQ had already been established elsewhere using valid methods. ${ }^{1}$

The DSM-IV diagnoses were based on current and retrospective assessment by a multidisciplinary team of experienced clinicians. For a diagnosis of ASD, semistructured clinical interviews based on the Autism Diagnostic Interview Revised (ADI-R) were used (Lord et al. 1994) as well as DSM-IV checklists, and all available information from schools and child psychiatric services with regard to the developmental history in childhood.

\footnotetext{
${ }^{1}$ Previous version of the WAIS (Dutch), or Groninger Intelligence Test (GIT).
} 
ADHD diagnoses were made according to a national protocol including a semi-structured developmental history and a DSM-IV criteria checklist for adult and childhood ADHD symptoms. Further details on the diagnostic procedure have been published earlier (Sizoo et al. 2009). The AQ was not part of the diagnostic process.

\section{Materials}

The AQ was translated into Dutch by a bi-lingual Dutch native speaker. The 50 items of the AQ are formulated in such a way that 24 statements confirm, and the other 26 deny ASD characteristics. The raw scores are recoded such that a higher score represents a higher degree to which the item is suggestive of ASD. These scores are then dichotomized $(1,2=0 ; 3,4=1)$ reflecting whether or not an ASD symptom is present (Woodbury-Smith et al. 2005). This yields a maximum possible total score of 50. A cut-off value of 26 has been suggested for a clinical population and a cut-off value of 32 for the general population (BaronCohen et al. 2001; Woodbury-Smith et al. 2005). Internal consistency of the total score of the translated test was good for the total group (Cronbach's $\alpha=0.81$ ), and for the ASD group (Cronbach's $\alpha=0.80$ ), but poor for the ADHD group (Cronbach's $\alpha=0.66$ ) (Cicchetti 1994).

\section{Statistics}

We used linear regression analysis to identify possible confounders (age, sex, or total IQ) in the relationship between diagnosis and AQ scores. We defined a (group of) variable(s) to be a confounder if entering that (group of) variable(s) into the model with diagnosis as independent and the AQ score as dependent, resulted in more than $10 \%$ change in the regression coefficient of diagnosis.

Assumptions for parametric tests were met and total AQ scores and subscales scores were analysed in ANOVA's with Diagnosis (ASD or ADHD) and SUD status (SUD-, SUD ${ }^{\wedge}$ and SUD+) as between subject factors. Partial eta squared in these analyses reflected the proportion of total variability attributable to each factor. Cut-off scores, sensitivity, specificity and percentage correctly classified patients were calculated using receiver operating characteristic (ROC) curves.

All statistical analyses were carried out with SPSS 15.0 software, using two-tailed tests with $\alpha=0.05$.

\section{Results}

\section{Clinical Characteristics}

The 129 subjects enrolled in the present study were adult Caucasian males $(n=97)$ and females $(n=32)$ (Table 1$)$.
The ASD group consisted of 76 subjects with a diagnosis Autistic Disorder $(n=10)$, Asperger Syndrome $(n=32)$, or Pervasive Developmental Disorder not otherwise specified (PDD-NOS: $n=34$ ). The distribution of these subtypes is comparable to other studies (Fombonne 2005 p.53). The ADHD group consisted of 53 subjects, 44 with the combined subtype, and 9 with the inattentive subtype. Substance use status was determined using DSM-IV criteria. Subjects with current abuse or dependence were designated as SUD+ $(n=28)$, and those with no life-time history of SUD as SUD- $(n=76)$. Subjects with a former history of SUD, who had been abstinent for at least 6 months prior to participation in the study, were designated as $\operatorname{SUD}^{\wedge}(n=25)$. Because behavioral addictions, like gambling, seem to share the same neurobiological underpinnings of craving and dependence, gambling was also included in our definition of SUD (Goudriaan et al. 2006; Potenza 2007).

\section{Group Characteristics and Types of SUD}

The ADHD and ASD groups were comparable with respect to IQ and age (Table 1). The percentage of female participants was significantly higher in the ADHD compared to the ASD group (34 vs. $18 \% ; X_{(1)}^{2}=4.043, p=0.044$ ). The percentage of lifetime SUD in the ADHD group was significantly higher than in the ASD group (60 vs. 28\%; $\left.X_{(1)}^{2}=13.83, p<0.001\right)$. In the ASD group with lifetime SUD, alcohol was the most frequent comorbid SUD, whereas other drugs and gambling accounted for most of the comorbid problems in the ADHD group with lifetime SUD.

\section{Effects of Diagnosis and SUD Status}

Since age, sex, and total IQ (independently or in combination) did not confound the main effect of Diagnosis on the AQ (sub) scores (see Statistics section). We did not correct for age, sex, or total IQ.

A statistically significant diagnosis by SUD interaction was found for the Social skill subscale (Table 2). Post hoc ANOVA analysis indicated that for this subscale the AQ score in the ASD group differed significantly depending on the SUD status $\left(F_{2}=3.158, p=0.05\right)$. The AQ score was significantly higher (indicating more problems with social skills) in the ASD subgroup without a history of SUD compared to the ASD subgroups with current SUD. There was no difference in social skill score among the SUD subgroups in the ADHD group.

The ASD group scored significantly higher compared to the ADHD group on the total AQ score, and on all but one of the subscales, namely Attention to detail. The SUD status did not significantly affect the AQ (sub-)scores. 
Table 1 Group characteristics and types of substance use disorder in adults with autism spectrum disorder or ADHD

\begin{tabular}{|c|c|c|c|c|c|}
\hline & \multicolumn{2}{|c|}{$\operatorname{ASD}(N=76)$} & \multicolumn{2}{|c|}{$\operatorname{ADHD}(N=53)$} & \multirow[t]{2}{*}{ Test statistic } \\
\hline & $N(\mathrm{pct})^{\mathrm{a}}$ & Mean (SD) & $N(\mathrm{pct})$ & Mean (SD) & \\
\hline Age & & $34.1(11.9)$ & & $32.1(11.4)$ & $t_{(127)}=0.960, p=0.339$ \\
\hline Female & $14(18)$ & & $18(34)$ & & $X_{(1)}^{2}=4.043, p=0.044$ \\
\hline Total IQ & & $103.0(13.6)$ & & $104.0(10.5)$ & $t_{(85)}=-0.364, p=0.717$ \\
\hline Lifetime SUD & $21(28)$ & & $32(60)$ & & $X_{(1)}^{2}=13.830, p<0.001$ \\
\hline Alcohol & $10(47)$ & & $8(25)$ & & $X_{(1)}^{2}=2.892, p=0.089$ \\
\hline Cannabis & $6(29)$ & & $9(28)$ & & $X_{(1)}^{2}=0.001, p=0.972$ \\
\hline Other drugs or gambling & $5(24)$ & & $15(47)$ & & $X_{(1)}^{2}=2.871, p=0.090$ \\
\hline
\end{tabular}

${ }^{\text {a }}$ Figures in $N$ column represent frequency (percent)

\section{Discriminating Power of the AQ}

A ROC analysis was conducted to assess the discriminant power, assuming that in using the AQ to discriminate between ADHD and ASD, it is equally important to minimize the false negatives, as it is to minimize the false positives. This optimum was established by determining which cut-off score was associated with the highest value of the sum of ASD screen positives and ADHD screen negatives. The cut-off score 26 resulted in a sensitivity of 0.68 , a specificity of 0.79 , and the correct classification of the greatest numbers in our population $(73 \%)$, which consisted for $59 \%$ of ASD patients. For our sample this meant that, using the optimum cut-off of 26, we would still fail to identify 24 out of 76 patients with ASD, and 11 out of 53 patients with ADHD. The associated false negative rate for ASD is 0.32 . We repeated the ROC computations for our population with and without SUD. With the cut-off at 26, the percentage correctly identified patients was not significantly different between the subgroups: without lifetime SUD $71 \%$, and with lifetime SUD $75 \%$.

\section{Discussion}

The aim of this study was to assess the usefulness of the AQ in differentiating between adult ASD and adult ADHD and to explore whether SUD comorbidity affects the AQ scores and hence the discriminant power of the AQ when using it in patient populations with SUD comorbidity.

This study indicates that the total AQ score and most of the AQ subscale scores (except the subscale score Attention to details) are significantly and substantially higher in adults with ASD compared to adults with ADHD, and that this effect is independent from SUD status. This is an important finding because it implies that the AQ might be a valid instrument in the differential diagnosis of ASD versus ADHD. This adds to the findings in previous studies, showing that the AQ differentiates between subjects with ASD and (comorbid) obsessive compulsive disorder or social anxiety disorder (Cath et al. 2008; Hoekstra et al. 2008). Examination of the two AQ-factors that were proposed by Hoekstra et al. (Hoekstra et al. 2008), shows that only the factor Social interaction accounts for the difference in AQ scores between ASD and ADHD subjects, but not the second factor Attention to details. The latter is composed of items referring to a perceptual style with a preference for details and patterns. We suggest that this scale does not discriminate between ASD and ADHD patients because it refers to a strategy for dealing with aspects of attention deficit that is common to both disorders. For example, it is known that people with ASD and ADHD present with comparable deficits in visual and auditory attention. Subjective reports from patients suggest that the attention deficit causes distress because they are overwhelmed by perceptual stimuli. The distress is said to reduce, when focusing on logical sequences such as patterns, telephone numbers or car license plates. In other words, the AQ differentiates between ASD and ADHD with respect to social interaction, as can be expected given that social interaction is the core deficit in ASD. People with ASD and ADHD show similarities, however, in the way they cope with attentional problems.

The second aim was to examine if the discriminating power of the AQ was negatively influenced if subjects have a comorbid SUD. The results show that this is only the case for the subscale Social skill in the ASD group, but not in the ADHD group. For all the other subscales, it appears that present or former SUD has no effect on the AQ scores, compared to subjects without a history of SUD. Our hypothesis is that comorbid SUD in ASD (mostly alcohol related in this group) improves or facilitates social skills and therefore social interaction. Alternatively, in people with ASD, alcohol may positively influence the subjective perception of their social skills, or cause a reduced awareness of social awkwardness, without actually improving their social functioning. 


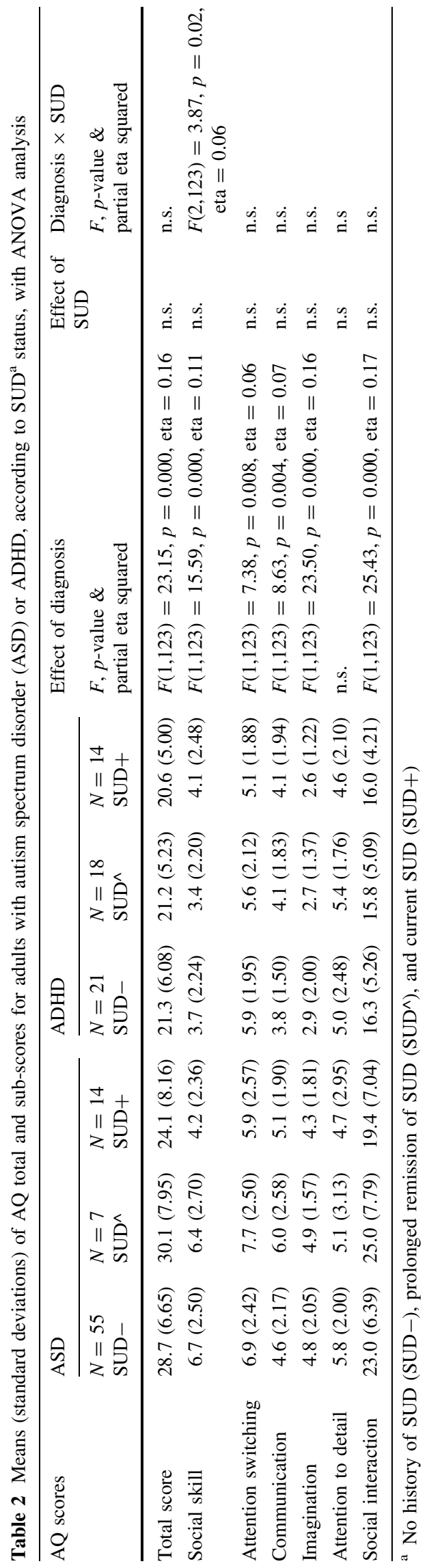

In sum, the AQ can differentiate between ASD and ADHD with statistical significance for the total AQ score and all subscales, with the exception of the subscale Attention to detail. However, when there is comorbidity with SUD the clinician should be cautious; in that case the total AQ score in patients with ASD can be attenuated by a lower score on the Social skills subscale.

The ROC analysis suggests a cut-off value of 26 , which yields $73 \%$ correctly identified patients. However, with this cut-off we would wrongly classify about a third of all ASD patients and a fifth of all ADHD patients in our study group. In the general population, the ratio of ADHD versus ASD prevalence is about 7:1, unlike in our sample where this ratio was about $2: 3$. The values for sensitivity and specificity associated with the cut-off score of 26 are, however, by definition independent of the prevalence. This means that, given the prevalences of ASD and ADHD in the general population, this cut-off would (in a hypothetical group of 50 patients) by approximation lead to a positive identification of 4 out of every 6 patients with ASD, and 35 out of every 44 patients with ADHD (that is $77 \%$ correctly identified patients). Clinical usefulness of the AQ for differentiating between ASD and ADHD is, therefore, limited because it would lead to an unacceptable number of wrongly classified patients. This underscores the continued necessity of other methods, like thorough clinical interviews, and eliciting an accurate developmental history, for distinguishing between a diagnosis of ASD and ADHD.

The data also show that the factor score Attention to detail is similar in the ASD and ADHD groups. If we were to use the other factor score Social interaction (with 40 items composed of the 4 remaining subscales), and use the associated optimum cut-off of 21 , the percentage correctly identified cases is almost the same (75\%) as in the case of the total AQ score. This indicates that the discriminating effect doesn't improve when we opt for this factor score instead of the total AQ score.

This study has both strengths and limitations. The main strengths are the relatively large sample size and the structured assessment of ASD and ADHD as well as the comorbid SUD's and pathological gambling. The main limitations are the unknown representativeness of this treatment seeking sample with its normal IQ, the absence of another psychiatric control group, and the relatively small size of some of the diagnostic subgroups. The sex distribution was similar to that of ASD and ADHD patients in epidemiological samples. However, the number of subjects with ASD exceeded those with ADHD, whereas the prevalence of ADHD in the general population is much higher than that of ASD (Gillberg and Wing 1999; Murphy and Barkley 1996). A possible explanation is that the participating expert centers are used more often by referral 
sources when ASD is suspected than when ADHD is suspected, because ADHD is more readily diagnosed and treated in general adult psychiatric settings.

Although the group of adults with ASD shows significantly higher scores on the Autism Spectrum Questionnaire (AQ) compared to group of adults with ADHD, the clinical usefulness of the instrument for the differentiation between ASD and ADHD in individual cases is limited. The established optimum cut-off value of 26 is similar to cutoff values previously reported by others, but the percentage of correctly identified patients with ASD or ADHD is only 73\%. Evidently, this self-report questionnaire cannot replace the clinical interview in disentangling ASD from ADHD.

Substance use disorder is a common comorbid problem accompanying ASD and ADHD. The results show that in case of comorbid SUD, adults with ASD still have significantly higher total AQ scores compared to adults with ADHD, even though SUD in people with ASD may present with a lower score on the subscale Social skills.

Acknowledgments We sincerely thank all participating patients for their enthusiastic contributions to the research project. We are grateful for the Dimence Institute of Mental Health management for their given support.

Open Access This article is distributed under the terms of the Creative Commons Attribution Noncommercial License which permits any noncommercial use, distribution, and reproduction in any medium, provided the original author(s) and source are credited.

\section{References}

American Psychiatric Association. (2000). Diagnostic and statistical manual of mental disorders (DSM IV). (4th text revised ed.) APA.

Anckarsäter, H., Stahlberg, O., Larson, T., Hakansson, C., Jutblad, S. B., Niklasson, L., et al. (2006). The impact of ADHD and autism spectrum disorders on temperament, character, and personality development. The American Journal of Psychiatry, 163, 1239-1244. doi:10.1176/appi.ajp.163.7.1239.

Baron-Cohen, S., Wheelwright, S., Skinner, R., Martin, J., \& Clubley, E. (2001). The Autism-spectrum quotient (AQ): Evidence from Asperger syndrome/high-functioning autism, males and females, scientists and mathematicians. Journal of Autism and Developmental Disorders, 31, 5-17. doi:10.1023/A:1005653411471.

Cath, D. C., Ran, N., Smit, J. H., van Balkom, A. J., \& Comijs, H. C. (2008). Symptom overlap between autism spectrum disorder, generalized social anxiety disorder and obsessive-compulsive disorder in adults: A preliminary case-controlled study. Psychopathology, 41, 101-110. doi:10.1159/000111555.

Cicchetti, D. V. (1994). Guidelines, criteria and rules of thumb for evaluating normed and standardized assessment instruments in psychology. Psychological Assessment, 6, 284-290. doi:10.1037/ 1040-3590.6.4.284.

Corbett, B. A., \& Constantine, L. J. (2006). Autism and attention deficit hyperactivity disorder: Assessing attention and response control with the integrated visual and auditory continuous performance test. Child Neuropsychology, 12, 335-348. doi: 10.1080/09297040500350938.

Courchesne, E., Lincoln, A. J., Yeung-Courchesne, R., Elmasian, R., \& Grillon, C. (1989). Pathophysiologic findings in nonretarded autism and receptive developmental language disorder. Journal of Autism and Developmental Disorders, 19, 1-17. doi: 10.1007/BF02212714.

Faraone, S. V., Biederman, J., Spencer, T., Mick, E., Murray, K., Petty, C., et al. (2006). Diagnosing adult attention deficit hyperactivity disorder: are late onset and subthreshold diagnoses valid? The American Journal of Psychiatry, 163, 1720-1729. doi:10.1176/appi.ajp.163.10.1720.

Fombonne, E. (2005). Epidemiological Studies in Pervasive Developmental Disorders. In F. R. Volkmar, R. Paul, A. Klin, \& D. Cohen (Eds.), Handbook of Autism and Pervasive Developmental Disorders (pp. 42-69). Hoboken: Wiley.

Gillberg, C., \& Wing, L. (1999). Autism: not an extremely rare disorder. Acta Psychiatrica Scandinavica, 99, 399-406. doi: 10.1111/j.1600-0447.1999.tb00984.x.

Goldstein, R. Z., \& Volkow, N. D. (2002). Drug addiction and its underlying neurobiological basis: neuroimaging evidence for the involvement of the frontal cortex. The American Journal of Psychiatry, 159, 1642-1652. doi:10.1176/appi.ajp.159.10. 1642.

Goudriaan, A. E., Oosterlaan, J., de Beurs, E., \& van den Brink, W. (2006). Psychophysiological determinants and concomitants of deficient decision making in pathological gamblers. Drug and Alcohol Dependence, 84, 231-239. doi:10.1016/j.drugalcdep. 2006.02.007.

Hoekstra, R. A., Bartels, M., Cath, D. C., \& Boomsma, D. I. (2008). Factor structure, reliability and criterion validity of the autismspectrum quotient (AQ): a study in Dutch population and patient groups. Journal of Autism and Developmental Disorders, 38, 1555-1566. doi:10.1007/s10803-008-0538-x.

Jensen, V. K., Larrieu, J. A., \& Mack, K. K. (1997). Differential diagnosis between attention-deficit/hyperactivity disorder and pervasive developmental disorder-not otherwise specified. Clinical Pediatrics, 36, 555-561. doi:10.1177/000992289703 601001.

Ketelaars, C., Horwitz, E., Sytema, S., Bos, J., Wiersma, D., Minderaa, R., et al. (2008). Brief report: Adults with mild autism spectrum disorders (ASD): Scores on the autismspectrum quotient (AQ) and comorbid psychopathology. Journal of Autism and Developmental Disorders, 38, 176-180. doi: 10.1007/s10803-007-0358-4.

Kurita, H., Koyama, T., \& Osada, H. (2005). Autism-spectrum quotient-Japanese version and its short forms for screening normally intelligent persons with pervasive developmental disorders. Psychiatry and Clinical Neurosciences, 59, 490-496. doi:10.1111/j.1440-1819.2005.01403.x.

Lord, C., Rutter, M., \& Le Couteur, A. (1994). Autism diagnostic interview-revised: A revised version of a diagnostic interview for caregivers of individuals with possible pervasive developmental disorders. Journal of Autism and Developmental Disorders, 24, 659-685. doi:10.1007/BF02172145.

Murphy, K., \& Barkley, R. A. (1996). Attention deficit hyperactivity disorder adults: Comorbidities and adaptive impairments. Comprehensive Psychiatry, 37, 393-401. doi:10.1016/S0010-440X (96) $90022-X$.

Potenza, M. N. (2007). Impulsivity and compulsivity in pathological gambling and obsessive-compulsive disorder. Revista Brasileira de Psiquiatria (Sao Paulo, Brazil), 29, 105-106. doi:10.1590/ S1516-44462007000200004.

Sizoo, B. B., van der Brink, W., Gorissen-van Eenige, M., \& van der Gaag, R. J. (2009). Personality characteristics of adults with autism spectrum disorders or attention deficit hyperactivity 
disorder with and without substance use disorders. The Journal of Nervous and Mental Disease, (in press).

van Wijngaarden, P. J. M., van der Brink, W., Couwenberg, C., Heerschop, J., \& van der Gaag, R. J.(2009). Autism spectrum disorders and substance use disorders: An exploratory study. (submitted).

Wakabayashi, A., Baron-Cohen, S., Wheelwright, S., \& Tojo, Y. (2006). The Autism-spectrum quotient (AQ) in Japan: A crosscultural comparison. Journal of Autism and Developmental Disorders, 36, 263-270. doi:10.1007/s10803-005-0061-2.

Wechsler, D. (1997). WAIS III, Dutch version, technical manual. Lisse: Swets \& Zeitlinger.
Wing, L. (1997). The autistic spectrum. Lancet, 350, 1761-1766. doi: 10.1016/S0140-6736(97)09218-0.

Woodbury-Smith, M. R., Robinson, J., Wheelwright, S., \& BaronCohen, S. (2005). Screening adults for Asperger syndrome using the AQ: A preliminary study of its diagnostic validity in clinical practice. Journal of Autism and Developmental Disorders, 35, 331-335. doi:10.1007/s10803-005-3300-7.

World Health Organization. (2001). International classification of functioning, disability, and health (2nd ed.). Geneva: World Health Organization. 